\title{
PERTURBATIVE ANALYSIS OF THE METHOD OF PARTICULAR SOLUTIONS FOR IMPROVED INCLUSION OF HIGH-LYING DIRICHLET EIGENVALUES*
}

\author{
A. H. BARNETT ${ }^{\dagger}$
}

\begin{abstract}
The Dirichlet eigenvalue or "drum" problem in a domain $\Omega \subset \mathbb{R}^{2}$ becomes numerically challenging at high eigenvalue (frequency) $E$. In this regime the method of particular solutions (MPS) gives spectral accuracy for many domain shapes. It requires a number of degrees of freedom scaling as $\sqrt{E}$, the number of wavelengths on the boundary, in contrast to direct discretization for which this scaling is $E$. Our main result is an inclusion bound on eigenvalues that is a factor $O(\sqrt{E})$ tighter than the classical bound of Moler-Payne and that is optimal in that it reflects the true slopes of curves appearing in the MPS. We also present an MPS variant that cures a normalization problem in the original method, while evaluating basis functions only on the boundary. This method is efficient at high frequencies, where we show that, in practice, our inclusion bound can give three extra digits of eigenvalue accuracy with no extra effort.
\end{abstract}

Key words. particular solutions, eigenvalue, Dirichlet, drum, Laplacian, inclusion

AMS subject classifications. 35P15, 47A55, 65N35, 65N15

DOI. $10.1137 / 080724022$

1. Introduction and main results. Computing the eigenvalues and eigenmodes of the Laplace operator with homogeneous boundary condition (BC) in a bounded domain $\Omega \subset \mathbb{R}^{d}$ for $d=2,3, \ldots$ is a classical problem with a 150 -yearlong history; for reviews, see [30,43]. It has a wealth of applications in physics and engineering including the vibration of membranes (drums), resonances and modes of acoustic, electromagnetic, and optical cavities and waveguides, energy states of quantum particles, and data analysis [40]. We focus on the case of a Dirichlet BC, i.e., acoustically sound-soft walls. The spectrum $\sigma_{D}:=\left\{E_{j}\right\}_{j=1}^{\infty}$ and modes $\left\{\phi_{j}\right\}_{j=1}^{\infty}$ are defined by

$$
\begin{array}{rlr}
-\Delta \phi_{j} & =E_{j} \phi_{j} & \text { in } \Omega, \\
\phi_{j} & =0 \quad \text { on } \partial \Omega .
\end{array}
$$

Physically the eigenvalues can be interpreted as frequencies; we order them (including multiplicities) $E_{1}<E_{2} \leq E_{3} \leq \cdots$. We may choose orthonormal modes, that is, $\left(\phi_{i}, \phi_{j}\right)_{\Omega}:=\int_{\Omega} \bar{\phi}_{i} \phi_{j} d \mathbf{x}=\delta_{i j}$ for all $i, j=1,2, \ldots$, where $\delta$ is the Kronecker delta.

There has been a growing interest in the computationally demanding case of high eigenvalue (high mode number $j$ ), where many wavelengths span the domain. One example is dielectric microcavity lasers, where the use of asymmetric shapes has led to much higher output powers; to predict lasing [45], a large number of cavity resonances (which can be approximated by Dirichlet modes) are needed at $j>10^{3}$. More broadly, the field of "quantum chaos" (short-wavelength asymptotic study of modes of systems with chaotic ray dynamics) has blossomed in the last 20 years in both physics [22] (with applications such as atomic physics and quantum dots) and mathematics (e.g.,

\footnotetext{
*Received by the editors May 13, 2008; accepted for publication (in revised form) January 5, 2009; published electronically May 27, 2009. This work was supported in part by a Courant Instructorship at NYU and by NSF grant DMS-0507614.

http://www.siam.org/journals/sinum/47-3/72402.html

${ }^{\dagger}$ Department of Mathematics, Dartmouth College, Hanover, NH 03755 (ahb@math.dartmouth. edu).
} 
quantum ergodicity [47] and arithmetic manifolds). Numerical investigation has been a vital part of this endeavor $[1,4]$ and has led to discoveries such as "scars" of periodic ray orbits in the modes [25].

The method of particular solutions (MPS), also known as collocation or "point matching," uses basis functions that satisfy the Helmholtz equation (1.1) but not necessarily (1.2). It was originally used by Fox, Henrici, and Moler [16] to compute low eigenvalues of an L-shaped domain to 8 digit accuracy, and has been improved by many, including Kuttler and Sigillito [29], and recently by Betcke and Trefethen [5]. Much of the key early analysis of the MPS appeared in SINUM [16, 33, 41, 10]. For geometries with many corners, a domain decomposition version was pioneered by Descloux and Tolley [8], modified by Driscoll [9] to find eigenvalues of the well-known pair of Gordon-Wolpert-Webb isospectral drums [21] to high accuracy, and further improved by Betcke [6]. In physics, where the emphasis is on high frequencies, related methods of Heller [25, 26] and the very efficient "scaling method" of Vergini and Saraceno $[46,3,4]$ have enjoyed unrivaled success. In this regime the advantage of the MPS is that the basis size scales (in $d=2$ ) as the wavenumber $\sqrt{E}$ (e.g., see [2]), rather than $E$ as with finite difference or finite element methods. (This advantage is shared by boundary integral methods [1]).

In the MPS a solution to (1.1) is approximated by a linear combination of basis functions $\left\{\xi_{n}\right\}_{n=1, \ldots, N}$, each of which satisfies $-\Delta \xi_{n}=E \xi_{n}$ in $\Omega$ at some trial frequency parameter $E$ but which do not individually satisfy the Dirichlet BC. Popular basis functions are plane waves [26], corner-adapted Fourier-Bessel functions $[16,9,5,6]$, and fundamental solutions $[12,27,4]$. If a trial solution $u \in \operatorname{Span}\left\{\xi_{n}\right\}$ satisfies $u=0$ on $\partial \Omega$ while $u \neq 0$ in $\Omega$, then it must be a multiple of a mode $\phi_{j}$ for some $j$, and we must have $E=E_{j}$. In practice, this is rarely achieved exactly. However, by defining the boundary error measure (or "tension")

$$
t=t[u]:=\frac{\|u\|_{\partial \Omega}}{\|u\|_{\Omega}}
$$

where $\|u\|_{\partial \Omega}$ and $\|u\|_{\Omega}$ are the usual $L^{2}$-norms on the boundary and interior, respectively, we have the following inclusion bound from a theorem of Moler-Payne [33, 29]. Let $-\Delta u=E u$ in $\Omega$, then there exists an eigenvalue $E_{j}$ satisfying

$$
\frac{\left|E-E_{j}\right|}{E_{j}} \leq C_{\Omega}^{\prime} t[u]
$$

where $C_{\Omega}^{\prime}$ is a constant depending only on the domain. $\left(C_{\Omega}^{\prime}\right.$ is an a priori upper bound on $\|h\|_{\Omega} /\|h\|_{\partial \Omega}$ for harmonic functions $h$ [29], hence (1.4) is known as an a priori-a posteriori inequality [30].) Eigenvalues may then be identified by searching the frequency $(E)$ axis for local minima of the function

$$
t_{m}(E):=\min _{u \in \operatorname{Span}\left\{\xi_{n}\right\}} t[u],
$$

as shown in Figure 1.1. Similar curves appear in [5, 7, 11]. Equation (1.4) states that the eigenvalue accuracy which may be claimed is controlled by the size of the minimum found.

However, observations of the slopes of curves such as Figure 1.1 led the author to notice that the Moler-Payne bounds are not optimal at high $E$. It turns out that, for $E$ in the neighborhood of each eigenvalue $E_{j}$, the bounds can be made a factor of $\sqrt{E}$ tighter, as follows. 


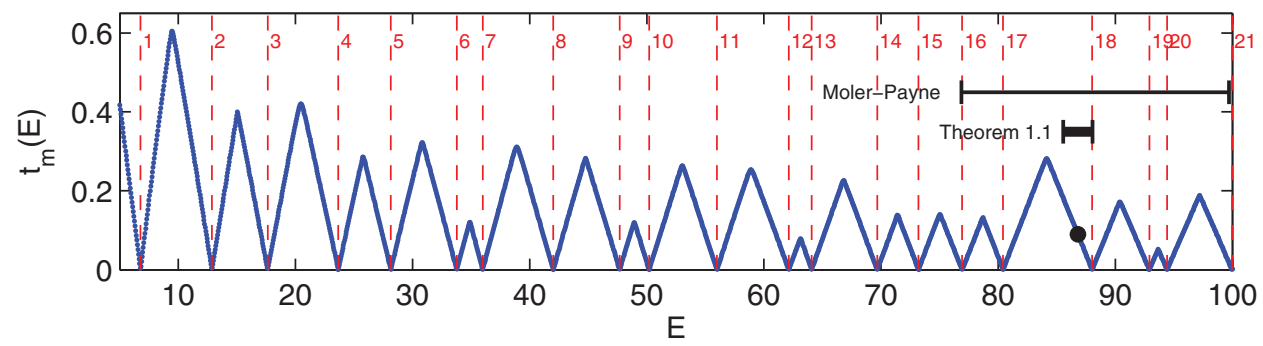

Fig. 1.1. Graph of $t_{m}(E)$ versus $E$ for the domain $(S)$ shown and defined in Figure 1.2 and weight $w=1$. Dirichlet eigenvalues $E_{j}$ are shown by vertical dotted lines labelled by $j$. At the single frequency $E=86.8$ (black dot), the inclusion bounds given by (1.4) (thin solid interval) and Theorem 1.1 with the choice $\epsilon=0.05$ (thick solid interval) are shown. The latter bounds are $\sqrt{E} \approx 10$ times tighter and optimal in the sense that they just include $E_{18}$ and correspond to the true slope of the $t_{m}(E)$ curve.

(S)
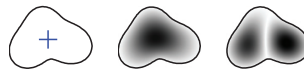

(T)
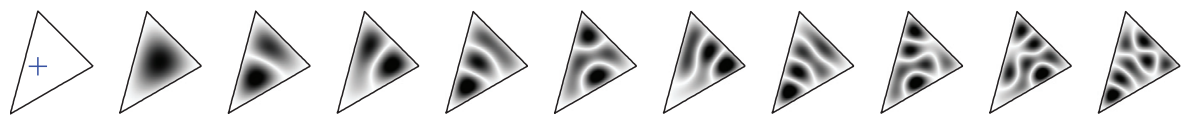

$(\mathrm{H})$
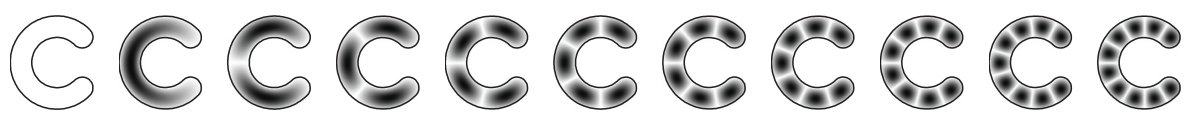

FIG. 1.2. Three domains used in verifying the utility of the main theorem and (right) their first ten eigenmodes (white is zero and darker represents larger absolute values $\left|\phi_{j}(\mathbf{x})\right|$ ). (S) smooth (in fact analytic) and strictly star-shaped, defined by the polar function $r(\theta)=1+0.2 \cos 2 \theta+$ $0.2 \cos (3 \theta+\pi / 3)$. (T) triangle, with angles $\pi / 4, \pi / 3$, and $5 \pi / 12$. (H) horseshoe, not star-shaped about any point, $C^{1}$-continuous boundary, the union of a $3 \pi / 2$ sector of the annulus of inner and outer radii 0.7 and 1.3 with two semicircles. In $(S)$ and $(T)$ the origin is shown by a + sign.

TheOREM 1.1. Let $\Omega \subset \mathbb{R}^{d}, d \geq 2$, be a smooth bounded domain. Fix $\epsilon>0$, then there is an open, possibly disconnected, set $\Sigma_{\epsilon} \subset(0, \infty)$ which contains the spectrum $\sigma_{D}$ and has the following property. For any $E \in \Sigma_{\epsilon} \backslash \sigma_{D}$ and any $u$ obeying $-\Delta u=E u$ in $\Omega$, there exists an eigenvalue $E_{j}$ satisfying

$$
\frac{\left|E-E_{j}\right|}{\sqrt{E_{j}}} \leq C_{\Omega}(1+\epsilon) t[u]
$$

where $C_{\Omega}$ is a constant depending on the domain but not on $\epsilon, \Sigma_{\epsilon}, E$, or $u$.

Comparing (1.6) to (1.4), we see that for high-frequency applications, the MPS is, in fact, more accurate, by a factor of the wavenumber, than has been realized before. Although the theorem does not indicate how large the set $\Sigma_{\epsilon}$ is (and therefore, how useful the bound is), we will show that in practice the set fills almost all of $(0, \infty)$ even for $\epsilon$ much smaller than 1. We explore this in section 4.2 for three example planar $(d=2)$ domains, and we demonstrate in section 4.3 that at $E \approx 10^{6}$ the bound on eigenvalue accuracy is improved by 3 digits without extra effort.

Remark 1.1. The error measure (1.3), a Rayleigh quotient, was used by the author in [3, Chapter 5] (as a special case, it is implicit in [29]) and cures a normalization problem inherent in the original MPS formulation [16]. This problem, and a very 
similar but independently found cure, is presented in [5]. Recently, Betcke [7] has united the various approaches within a single framework.

One of the main goals of this paper is to prove Theorem 1.1, which we do in sections 2 and 3 . This will rely on the following: Lemma 2.1 , which bounds $t[u]$ by a frequency (parameter) dependent eigenvalue of an operator $A(E)$ defined by $(2.3)$ acting in $L^{2}(\partial \Omega)$; Theorem 2.2, which describes the quadratic parameter-dependence of such eigenvalues in the neighborhood of each $E_{j}$; and Lemma 2.3, an upper bound on the norm of eigenmode normal derivatives on the boundary. A deeper purpose of our work is to understand the structure of MPS curves such as Figures 1.1 and 5.1. We believe this can lead to better numerical methods and are particularly motivated by the variants of such curves which form the heart of the scaling method [46, 3, 4]. Section 3, where we apply perturbation theory in the frequency parameter to prove Theorem 2.2 , is a step in this direction. We actually do this for a more general boundary norm

$$
\|u\|_{w, \partial \Omega}:=\left(\int_{\partial \Omega} w|u|^{2} d s\right)^{1 / 2}
$$

where $w$ is some strictly positive bounded weight function on $\partial \Omega$ and $d s$ is the usual surface element. One point of introducing $w$ is that it allows the following useful special case, where $\mathbf{x}(s) \in \partial \Omega$ is position vector and $\mathbf{n}(s)$ the outwards unit normal.

Proposition 1.2. Let $\Omega$ be strictly star-shaped about the origin, and let $w=$ $(\mathbf{x} \cdot \mathbf{n})^{-1}$. If we replace the norm $\|u\|_{\partial \Omega}$ by $\|u\|_{w, \partial \Omega}$ in (1.3), then in Theorem 1.1 we may choose the constant $C_{\Omega}=\sqrt{2}$, independent of $\Omega$ or $d$.

Note that this geometric restriction on $\Omega$ is needed if $w$ is to be positive and bounded. We demonstrate the utility of this explicit bound in section 4 .

A secondary goal is to describe (in section 4.1) an implementation of the MPS, in particular, how to compute $t_{m}(E)$ using a generalized matrix eigenvalue problem and basis evaluations on the boundary alone. The latter makes our method more efficient than existing MPS methods $[29,5]$ at high frequency. Finally, we conclude in section 5 .

2. Parameter-dependent boundary operator and proof of the theorem. Consider the interior Dirichlet boundary-value problem (BVP) for the homogeneous Helmholtz equation at frequency $E$,

$$
\begin{aligned}
(\Delta+E) u & =0 & & \text { in } \Omega, \\
u & =U & & \text { on } \partial \Omega .
\end{aligned}
$$

We use small letters to indicate functions on $\Omega$ and the corresponding capital letter for their restriction to $\partial \Omega$ (boundary trace). Given $E \notin \sigma_{D}$, the BVP (2.1) with boundary data $U \in L^{2}(\partial \Omega)$ has a unique solution in $L^{2}(\Omega)$ [32, Theorem 4.25]. Henceforth, we work in a $w$-weighted inner product

$$
\langle U, V\rangle:=\int_{\partial \Omega} \bar{U} V w d s,
$$

where $0<c \leq w(s) \leq C$, for all $s \in \partial \Omega$ for some constants $c, C$. Thus (1.7) may be written $\sqrt{\langle U, U\rangle}$. The point of this weight is to allow choices such as that in Proposition 1.2, which may be more useful than the case $w=1$, which is common in the literature. 
The parameter-dependent operator $A=A(E)$ is defined by

$$
\langle U, A(E) V\rangle=(u, v)_{\Omega}, \quad \text { for all } U, V \in L^{2}(\partial \Omega) .
$$

(By $v$ we mean the unique interior solution to (2.1) with data $V$ ). A unique such operator in $L^{2}(\partial \Omega)$ exists by the Riesz representation theorem. By (2.3), it is positive and self-adjoint (in the $w$-weighted norm) for real $E$. Treating (1.4) as an upper bound on $\|u\|_{\Omega}$, it follows that $A(E)$ is bounded for all $E \notin \sigma_{D}$. An interpretation of $A$ is that its sesquilinear form encodes the domain inner product of the interior Helmholtz extensions of two given boundary functions.

An inverse eigenvalue $\lambda(E)$ of $A$ is defined at each $E \notin \sigma_{D}$ by

$$
A(E) X(E)=\frac{1}{\lambda(E)} X(E)
$$

with corresponding eigenfunction $0 \neq X(E) \in L^{2}(\partial \Omega)$. We are interested in how the (inverse) spectrum of $A(E)$ depends on $E$. At this point we might stop to ask whether $A$ even possesses a point spectrum. (2.1) has a solution operator $\mathcal{K}$ which maps $U$ to $u$. For smooth domains $\left(C^{\infty}\right.$ boundary), $\mathcal{K}$ is bounded from $L^{2}(\partial \Omega)$ to $H^{1 / 2}(\Omega)$ (e.g., see [31]), thus by standard Sobolev embedding theorems, is compact from $L^{2}(\partial \Omega)$ to $L^{2}(\Omega)$. Since $A=\mathcal{K}^{*} \mathcal{K}$, where $\mathcal{K}^{*}$ is the adjoint with respect to the appropriate inner products, $A$ is compact. Thus $A$ has pure point spectrum, and the sequence $\left\{\lambda_{i}(A)\right\}_{i=1,2, \ldots}$ has no accumulation point. If $\Omega$ has corners, we expect (and have numerical evidence) that $A$ remains compact but do not yet know a proof.

The $w$-weighted version of (1.3) is (we retain the same symbol)

$$
t[u]:=\frac{\|u\|_{w, \partial \Omega}}{\|u\|_{\Omega}} .
$$

For general $w$, we have the following simple lower bound on $t[u]$ in terms of the parameter-dependent spectrum of $A(E)$.

Lemma 2.1. Let $\lambda_{1}(E)$ be the lowest inverse eigenvalue of $A(E)$ defined by (2.4). Fix $E>0$, then for all $u$ obeying $-\Delta u=E u$ in $\Omega$, it holds that

$$
t[u] \geq \sqrt{\lambda_{1}(E)},
$$

where $t[u]$ is defined by $(2.5)$.

Proof. Since $A$ is self-adjoint and bounded,

$$
\|u\|_{\Omega}^{2}=\langle U, A U\rangle \leq \frac{1}{\lambda_{1}}\|U\|_{w, \partial \Omega}^{2},
$$

and the proof follows from the definition (2.5).

We now describe the behavior of this lowest inverse eigenvalue for parameter values $E$ in the neighborhood of the Dirichlet spectrum of $\Omega$. We will make frequent use of the weighted eigenmode boundary functions

$$
\psi_{j}:=\frac{1}{w} \partial_{n} \phi_{j},
$$

where $\partial_{n} \phi_{j}:=\left.\mathbf{n} \cdot \nabla \phi_{j}\right|_{\partial \Omega}$ is the usual normal derivative. These turn out to be the natural boundary representation of modes associated with the norm (1.7). We also need the infinite matrix $Q=\left(Q_{i j}\right)$ whose entries are the weighted mode boundary inner products

$$
Q_{i j}:=\left\langle\psi_{i}, \psi_{j}\right\rangle, \quad i, j=1,2, \ldots
$$

The proof of the following theorem is more involved and is the purpose of section 3 . 
Theorem 2.2. Fix $j \in \mathbb{N}$, and let $p$ be the (finite) multiplicity of $E_{j}$. Without loss of generality choose $j$ to be the first in the list of degenerate eigenvalues, that is, $E_{j}=E_{j+1}=\cdots=E_{j+p-1}$. Then in the limit as $E$ tends to $E_{j}$, precisely $p$ inverse eigenvalues of $A(E)$ vanish in the following fashion:

$$
\lambda_{i}(E)=c_{j}^{(i)}\left(E-E_{j}\right)^{2}+O\left(\left(E-E_{j}\right)^{4}\right), \quad i=1, \ldots, p,
$$

where the curvature coefficients $c_{j}^{(1)} \leq c_{j}^{(2)} \leq \cdots \leq c_{j}^{(p)}$ are given by the inverse eigenvalues of the $p \times p$ submatrix $\tilde{Q}:=\left(Q_{i k}\right)_{i, k=j, \ldots, j+p-1}$. The smallest coefficient satisfies

$$
\frac{1}{c_{j}^{(1)}}=\sup _{\phi \in \Phi,\|\phi\|_{\Omega}=1} \int_{\partial \Omega} \frac{1}{w}\left|\partial_{n} \phi\right|^{2} d s
$$

where $\Phi:=\operatorname{Span}\left\{\phi_{j}, \ldots, \phi_{j+p-1}\right\}$ is the $E_{j}$-eigenspace.

This locally quadratic behavior of the smallest inverse eigenvalue $\lambda_{1}(E)$ versus frequency $E$ is visible in Figure 1.1: $t_{m}(E)$ is a good approximation to $\sqrt{\lambda_{1}(E)}$ and appears locally linear. Note that when $E_{j}$ is a simple eigenvalue $(p=1)$, this gives

$$
\frac{1}{c_{j}^{(1)}}=\left\|\psi_{j}\right\|_{w, \partial \Omega}^{2}=\int_{\partial \Omega} \frac{1}{w}\left|\partial_{n} \phi_{j}\right|^{2} d s .
$$

The right-most expressions in (2.11) and (2.12) are now bounded above because of the following, which puts a limit on how "shallow" the parabola $\lambda_{1}(E)$ may be.

LEMma 2.3 (Rellich). Given a bounded Lipschitz domain $\Omega \subset \mathbb{R}^{d}$, there are constants $C_{w, \Omega} \geq c_{w, \Omega}>0$ depending only on $\Omega$ and the weight $w$, such that

$$
c_{w, \Omega}^{2} E_{j} \leq \int_{\partial \Omega} \frac{1}{w}\left|\partial_{n} \phi_{j}\right|^{2} d s \leq C_{w, \Omega}^{2} E_{j} \quad \text { for all } j=1,2, \ldots
$$

For the special case $w=(\mathbf{x} \cdot \mathbf{n})^{-1}$, one may choose $c_{w, \Omega}=C_{w, \Omega}=\sqrt{2}$, independent of $\Omega$, in which case the inequalities become an equality.

The inequalities are proved in Hassell-Tao [24, section 2] in the case $w=1$ and $\partial \Omega$ smooth but carry over trivially to strictly positive bounded $w$. The lower bound uses (2.14) below, thus holds for Lipschitz domains. They derived the upper bound using commutator estimates involving a smooth vector field which points normally outwards on $\partial \Omega$. (Also see [19, Lemma 2.1] for the case of domains with a Lipschitz normal vector field.) A simple generalization of this vector field construction to be smooth with merely positive normal component on $\partial \Omega$ allows the upper bound to carry over to Lipschitz domains. For instance, the vector field $\mathbf{x}$ works for strictly star-shaped Lipschitz domains [23]. The special-weight case in Lemma 2.3 is due to Rellich [38], who proved

$$
\int_{\partial \Omega} \mathbf{x} \cdot \mathbf{n}\left|\partial_{n} \phi\right|^{2} d s=2 E_{j}
$$

for any bounded $\Omega$ and dimension $d$ (regardless of whether $\Omega$ is star-shaped).

Remark 2.1. The argument and Remark of [24, section 2] carries over to the case of $\Omega$ possessing a boundary of only $C^{1}$ continuity, since the first derivatives of the constructed vector field remain bounded. Therefore, with $w=1$, we get that $C_{w, \Omega}$ is the inverse of the largest $\delta$ such that the function $r(\mathbf{x})=\operatorname{dist}(\mathbf{x}, \partial \Omega), \mathbf{x} \in \Omega$, is $C^{1}$ continuous wherever $r \in[0, \delta]$. For domain (H) of Figure 1.2, this gives $C_{w, \Omega}=10 / 3$, the inverse of the minimum radius of curvature. 
2.1. Proof of main theorem. We now have all the pieces to prove Theorem 1.1, more precisely, its generalization to general strictly positive bounded weight $w$. For a given eigenmode number $j$, Theorem 2.2 implies that $\lim _{E \rightarrow E_{j}} c_{j}^{(1)}\left(E-E_{j}\right)^{2} / \lambda_{1}(E)=$ 1 , thus there is a $\delta_{j}>0$ such that

$$
\sqrt{\frac{c_{j}^{(1)}}{\lambda_{1}(E)}}\left|E-E_{j}\right| \leq 1+\epsilon
$$

holds for all $E$ in the punctured interval $0<\left|E-E_{j}\right|<\delta_{j}$. By choosing $\Sigma_{\epsilon}=$ $\bigcup_{j=1}^{\infty}\left\{E:\left|E-E_{j}\right|<\delta_{j}\right\}$, then for each $E \in \Sigma_{\epsilon} \backslash \sigma_{D}$, there exists a $j$ such that (2.15) holds. Substituting into (2.15), the bound from Lemma 2.1 and the upper bound in Lemma 2.3 completes the proof of Theorem 1.1, with the constant $C_{\Omega}$ being equal to the upper constant $C_{w, \Omega}$ in Lemma 2.3 .

Remark 2.2. Theorem 2.2 is stronger than necessary for this proof, since only the existence of $\lim _{E \rightarrow E_{j}} c_{j}^{(1)}\left(E-E_{j}\right)^{2} / \lambda_{1}(E)$ is needed. In fact, the full analyticity of $\lambda_{1}(E)$ will be proved in section 3 .

3. Proof of quadratic behavior of inverse eigenvalues of $\boldsymbol{A}$. Here (with Appendices A and B) we prove Theorem 2.2. The first part involves a modal expansion, which allows us to split the operator $A(E)$ into a finite-rank part associated with the $j$ th eigenspace, and a remainder, which we show is analytic in the parameter $E$ in the neighborhood of $E_{j}$. We then apply Rellich-Kato analytic operator perturbation theory to show that the inverse eigenvalues $\left\{\lambda_{i}(E)\right\}_{i=1, \ldots, p}$ have quadratic behavior about $E_{j}$. However, there is a twist: the needed eigenvalue(s) of $A(E)$ have poles at $E=E_{j}$. This we may cancel by scalar multiplication, but the price to pay is that analytic perturbation theory is no longer applicable to the resulting infinite multiplicity zero eigenvalue. Nevertheless, the Cauchy interlacing property will recover what is needed.

3.1. Mode expansion of the boundary operator. The key to our proof is to express our boundary operator $A$ as an infinite sum of rank-1 operators associated with each eigenmode boundary function.

LEMMA 3.1.

$$
A(E)=\sum_{j=1}^{\infty} \frac{\psi_{j}\left\langle\psi_{j}, \cdot\right\rangle}{\left(E-E_{j}\right)^{2}} .
$$

Remark 3.1. A similar formula (for the case $w=1$ ) is known [34] for the Dirichletto-Neumann map for (2.1). In fact, when $w=1$ it can be shown directly that $-A(E)$ is the $E$-derivative of the Dirichlet-to-Neumann map [17, equation (2.5)].

Proof. The Helmholtz equation Green's function $G$ in the domain $\Omega$ is defined by [18]:

$$
\begin{aligned}
\left(-\Delta_{\mathbf{x}}-E\right) G(\mathbf{x}, \mathbf{y}) & =\delta(\mathbf{x}-\mathbf{y}) & & \text { for } \mathbf{x}, \mathbf{y} \in \Omega, \\
G(\mathbf{x}, \mathbf{y}) & =0 & & \text { for } \mathbf{x} \in \partial \Omega \text { or } \mathbf{y} \in \partial \Omega .
\end{aligned}
$$

$G$ depends on $E$, although we shall not indicate this explicitly. $\delta$ is the Dirac delta distribution in $\mathbb{R}^{d}$. Then the solution operator $\mathcal{K}$ for the BVP (2.1) is

$$
u(\mathbf{x})=(\mathcal{K} U)(\mathbf{x})=\int_{\partial \Omega} \mathcal{K}(\mathbf{x}, s) U(s) d s \quad \text { for } \mathbf{x} \in \Omega,
$$


where $s \in \partial \Omega$ is a boundary coordinate and the Poisson kernel for the Helmholtz equation is

$$
\mathcal{K}(\mathbf{x}, s)=\partial_{n(s)} G(\mathbf{x}, s),
$$

where here $\partial_{n(s)}$ indicates the normal derivative with respect to the second argument, evaluated at the boundary point $s$. From (3.3) we get

$$
(u, v)_{\Omega}=\int_{\partial \Omega} \int_{\partial \Omega} \overline{U(s)} V\left(s^{\prime}\right) \int_{\Omega} \partial_{n(s)} \overline{G(\mathbf{x}, s)} \partial_{n\left(s^{\prime}\right)} G\left(\mathbf{x}, s^{\prime}\right) d \mathbf{x} d s d s^{\prime} .
$$

Since (2.3) holds for arbitrary $U, V \in L^{2}(\partial \Omega)$, we may write the action of $A$ on any $V \in L^{2}(\partial \Omega)$ as the integral operator $(A V)(s)=\int_{\partial \Omega} A\left(s, s^{\prime}\right) V\left(s^{\prime}\right) d s^{\prime}$ whose kernel is

$$
A\left(s, s^{\prime}\right)=\frac{1}{w(s)} \int_{\Omega} \partial_{n(s)} \overline{G(\mathbf{x}, s)} \partial_{n\left(s^{\prime}\right)} G\left(\mathbf{x}, s^{\prime}\right) d \mathbf{x} .
$$

Inserting the usual eigenfunction expansion [18]

$$
G(\mathbf{x}, \mathbf{y})=\sum_{j=1}^{\infty} \frac{\overline{\phi_{j}(\mathbf{x})} \phi_{j}(\mathbf{y})}{E-E_{j}}
$$

into (3.6), orthonormality on $\Omega$ kills the domain integral and one of the sums, leaving

$$
A\left(s, s^{\prime}\right)=\frac{1}{w(s)} \sum_{j=1}^{\infty} \frac{\partial_{n} \overline{\phi_{j}(s)} \partial_{n} \phi_{j}\left(s^{\prime}\right)}{\left(E-E_{j}\right)^{2}} .
$$

The apparent lack of symmetry of the kernel is deceptive and is merely a result of the weighted inner product (2.2). It is simple to check that this kernel is equivalent to the desired symmetric formula (3.1).

Remark 3.2. Despite its suggestive form, (3.1) is not a sum of orthogonal projections, nor a spectral representation, since $\left\{\psi_{j}\right\}$ are not unit norm and, although complete in $L^{2}(\partial \Omega)$, they are generally not orthogonal.

Remark 3.3. Although each term in (3.1) is a bounded operator, with bound $C_{w, \Omega}^{2} E_{j} /\left(E-E_{j}\right)^{2}$ following from Lemma 2.3, the sum of these bounds is divergent for all $d \geq 2$. This follows from Weyl's law [18, Chapter 11], which states that asymptotically, the number of Laplacian eigenvalues below $E$ grows like $E^{d / 2}$. Therefore, (3.1) cannot directly be used to show that $A$ is the norm limit of finite-rank operators and hence compact.

3.2. Perturbation theory for inverse eigenvalues. From now on we fix the mode number $j$, with multiplicity $p$. Recall if $p>1$, then for convenience, $j$ is chosen to be the lowest in the degenerate set $j, \ldots, j+p-1$. It is a standard result that $p$ is finite, since the Laplacian has compact inverse [13, section 6.5.1]. We separate the terms in the sum (3.1) associated with this set, giving

$$
\left(E-E_{j}\right)^{2} A(E)=B_{j}+\left(E-E_{j}\right)^{2} A_{j}(E),
$$

where the fixed rank- $p$ operator associated with the $E_{j}$-eigenspace is

$$
B_{j}:=\sum_{m=1}^{p} \psi_{j+m-1}\left\langle\psi_{j+m-1}, \cdot\right\rangle
$$


and the remainder is a scalar multiple of the bounded operator

$$
A_{j}(E):=\sum_{m \in \mathbb{N}, m \notin[j, j+p-1]} \frac{\psi_{m}\left\langle\psi_{m}, \cdot\right\rangle}{\left(E-E_{m}\right)^{2}} .
$$

With respect to the parameter $E$, the latter operator has second-order poles at all eigenvalues not equal to $E_{j}$. It is also analytic near $E_{j}$ (the proof is in Appendix A).

LEMMA 3.2. $A_{j}(E)$ is a bounded regular (analytic) operator in the sense of Rellich $\left[39\right.$, p. 55] for $E$ in a neighborhood of $E_{j}$. That is, for each $f \in L^{2}(\partial \Omega)$, the element $A_{j}(E) f$ exists as a power series $f_{0}+\left(E-E_{j}\right) f_{1}+\left(E-E_{j}\right)^{2} f_{2}+\cdots$ convergent in $L^{2}(\partial \Omega)$ for $E$ in a neighborhood of $E_{j}$.

Now define $\left\{\mu_{i}(E)\right\}$ to be the set of parameter-dependent eigenvalues of the operator $\left(E-E_{j}\right)^{2} A(E)$. We wish to understand their behavior when $E$ is close to $E_{j}$, since from them we can simply recover the desired $\lambda(E)$ as defined in (2.4). We first characterize the eigenvalues of $B_{j}$ in terms of $\tilde{Q}$, the mode boundary inner product matrix for the eigenspace.

LEMMA 3.3. $B_{j}$ has (counting multiplicity) precisely $p$ nonzero eigenvalues $\nu_{1} \geq$ $\nu_{2} \geq \cdots \geq \nu_{p}$, which are identical to the set of eigenvalues (counting multiplicity) of the matrix $\tilde{Q}$ defined by Theorem 2.2 and (2.9). $B_{j}$ also has a zero eigenvalue of infinite multiplicity.

Proof. Given $\boldsymbol{\beta}:=\left(\beta_{1}, \ldots, \beta_{p}\right)^{T}$, define $\phi=\sum_{m=1}^{p} \beta_{m} \phi_{j+m-1}$, then by orthonormality $\|\phi\|_{\Omega}=|\boldsymbol{\beta}|$, the usual Euclidean norm in $\mathbb{C}^{p}$. Thus $\phi$ is an $L^{2}(\Omega)$-normalized eigenmode whenever $|\boldsymbol{\beta}|=1$. Its weighted normal derivative is $\psi=\frac{1}{w} \partial_{n} \phi$. Then, using (2.9) and (2.13) we have

$$
\inf _{|\boldsymbol{\beta}|=1} \boldsymbol{\beta}^{H} \tilde{Q} \boldsymbol{\beta}=\inf _{|\boldsymbol{\beta}|=1} \sum_{m, n=1}^{p} \overline{\beta_{m}} \beta_{n}\left\langle\psi_{j+m-1}, \psi_{j+n-1}\right\rangle=\|\psi\|_{w, \partial \Omega}^{2} \geq c_{w, \Omega}^{2} E_{j}>0
$$

Thus all eigenvalues of $\tilde{Q}$ are positive, and also the vectors $\left\{\psi_{j+m-1}\right\}_{m=1, \ldots, p}$ are linearly independent. From the definition (3.10) any eigenvector of $B_{j}$ with nonzero eigenvalue must lie in $\operatorname{Span}\left\{\psi_{j+m-1}\right\}_{m=1, \ldots, p}$. Suppose $\sum_{m=1}^{p} \beta_{m} \psi_{j+m-1}$ is such an eigenvector with eigenvalue $\nu$. Using (2.9), this is equivalent to the statement

$$
\sum_{m, n=1}^{p} \beta_{m} \psi_{n} \tilde{Q}_{m n}=\nu \sum_{n=1}^{p} \beta_{n} \psi_{n}
$$

By linear independence, we equate coefficients of $\psi_{n}$, so (3.13) is equivalent to $\tilde{Q} \boldsymbol{\beta}=$ $\nu \boldsymbol{\beta}$, and these two problems have the same set of nonzero eigenvalues. The infinite multiplicity zero eigenvalue exists, since $B_{j}$ has finite rank (degenerate kernel).

We would like to apply perturbation theory in the small parameter $\left(E-E_{j}\right)$ to (3.9). Unfortunately, its infinite multiplicity prevents the results of analytic perturbation theory from being applied directly to the zero eigenvalue of $B_{j}$. We circumvent this problem ${ }^{1}$ by appealing to the following classical property of the point spectrum under a rank-1 perturbation (for convenience, we include a proof in this operator setting in Appendix B).

LEMma 3.4 (Cauchy interlacing). Let $S$ be a self-adjoint operator in a Hilbert space, bounded from below, with eigenvalues $\sigma_{1} \leq \sigma_{2} \leq \cdots$. Let $\psi\langle\psi, \cdot\rangle$ define a

\footnotetext{
${ }^{1}$ The author is indebted to Percy Deift for this argument.
} 
(a)

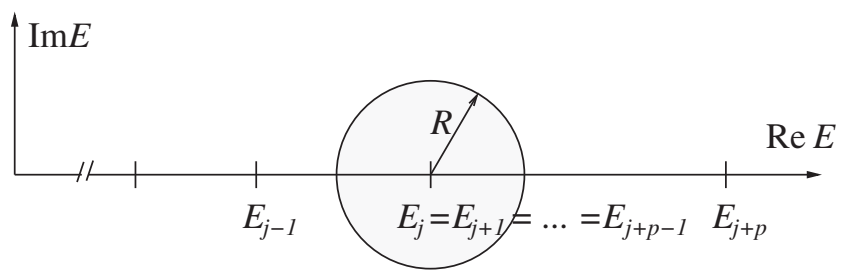

(b)

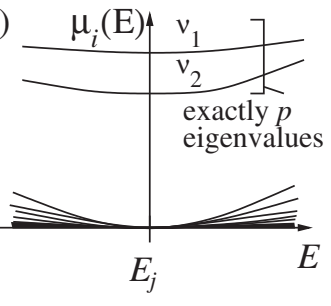

FIG. 3.1. (a) Complex E plane showing disc around the current eigenvalue $E_{j}$ used in the proof of Lemma 3.2. The operator $A(E)$ has second-order poles at each of the $E_{j}$. (b) Sketch of the spectrum $\left\{\mu_{i}(E)\right\}$ of the operator $\left(E-E_{j}\right)^{2} A(E)$ used to prove Theorem 2.2.

(nonnegative) rank-1 operator, and let the eigenvalues of $S-\psi\langle\psi, \cdot\rangle$ be $\tau_{1} \leq \tau_{2} \leq \cdots$. Then $\tau_{1} \leq \sigma_{1} \leq \tau_{2} \leq \sigma_{2} \leq \tau_{3} \leq \cdots$.

For real $E \neq E_{j}$ in the domain of analyticity of $A_{j}(E)$, we proceed inductively as follows. If $p=1$, this lemma applies with $S=-\left(E-E_{j}\right)^{2} A_{j}(E)$ and $\psi=\psi_{j}$. The result is that at most one eigenvalue $\mu_{1}(E)$ may exceed the largest eigenvalue of $\left(E-E_{j}\right)^{2} A_{j}(E)$. For $p>1$, we may apply the interlacing lemma $p$ times and conclude that at most $p$ eigenvalues $\mu_{1}, \mu_{2}, \ldots$ exceed the largest eigenvalue of $\left(E-E_{j}\right)^{2} A_{j}(E)$. The eigenvalues of $A_{j}(E)$ are analytic by the theorem of Rellich [39, Theorem II.2.1], which applies due to Lemma 3.2. Therefore, the eigenvalues of $\left(E-E_{j}\right)^{2} A_{j}(E)$ have convergent power series beginning with $\left(E-E_{j}\right)^{2}$ and hence vanish as $E \rightarrow E_{j}$. This leaves a set of at most $p$ eigenvalues $\mu_{i}(E)$, which may have nonzero limits as $E \rightarrow E_{j}$. This behavior is illustrated in Figure 3.1b. So we have proved that there is, after all, no complication introduced by the infinite multiplicity of the zero eigenvalue. Since by (3.9), $\left(E-E_{j}\right)^{2} A(E)$ is also bounded analytic in a neighborhood of $E_{j}$, the Rellich theorem tells us that members of the above-mentioned set $\mu_{i}(E)$ are analytic in a neighborhood of $E_{j}$, so at $E=E_{j}$ their limits must coincide with the set $\left\{\nu_{i}\right\}_{i=1, \ldots, p}$ of nonzero eigenvalues of $B_{j}$. Thus we have proved that there are $p$ eigenvalues of the operator $\left(E-E_{j}\right)^{2} A(E)$ behaving as

$$
\mu_{i}(E)=\nu_{i}+O\left(\left(E-E_{j}\right)^{2}\right), \quad i=1, \ldots, p .
$$

Note that there is no first-order term in $\left(E-E_{j}\right)$ because the perturbation term in (3.9) has a power series beginning with $\left(E-E_{j}\right)^{2}$.

Eigenvalues $\mu_{i}(E)$ and $\lambda_{i}(E)$ are connected for $E \neq E_{j}$ via (2.4) and (3.9), so

$$
\lambda_{i}(E)=\frac{\left(E-E_{j}\right)^{2}}{\mu_{i}(E)} .
$$

Combining this with (3.14) shows that there are precisely $p$ inverse eigenvalues of $A(E)$ which vanish as $E \rightarrow E_{j}$, proving the first part of Theorem 2.2:

$$
\lambda_{i}(E)=\frac{1}{\nu_{i}}\left(E-E_{j}\right)^{2}+O\left(\left(E-E_{j}\right)^{4}\right), \quad i=1, \ldots, p .
$$

Finally, we now place a lower bound on the curvature coefficient of the asymptotically smallest eigenvalue $\lambda_{1}(E)$. Comparing the above to (2.10) and using Lemma 3.3 yields $c_{j}^{(1)}=\nu_{1}^{-1}=\left(\sup _{|\boldsymbol{\beta}|=1} \boldsymbol{\beta}^{H} \tilde{Q} \boldsymbol{\beta}\right)^{-1}$. Noticing that $\boldsymbol{\beta}^{H} \tilde{Q} \boldsymbol{\beta}=\|\psi\|_{w, \partial \Omega}^{2}=$ $\int_{\partial \Omega} \frac{1}{w}\left|\partial_{n} \phi\right|^{2} d s$, where $\phi$ and $\psi$ are defined as in the proof of Lemma 3.3, completes the proof. 
4. Implementation and performance of eigenvalue inclusion. Here we discuss an efficient two-dimensional $(d=2)$ implementation of the MPS, then show that, in practice, Theorem 1.1 gives useful eigenvalue inclusion bounds even for $\epsilon$ much smaller than 1. As illustrated in Figure 1.1, these supercede the Moler-Payne bounds.

4.1. Computation of minimum boundary error $t_{m}(E)$. At each given frequency $E$, inserting the basis representation $u=\sum_{n=1}^{N} \alpha_{n} \xi_{n}$ converts the minimum boundary error (1.5) into the Rayleigh quotient (here, $\boldsymbol{\alpha}:=\left(\alpha_{1}, \ldots, \alpha_{N}\right)^{T}$ and ${ }^{H}$ indicates conjugate transpose)

$$
t_{m}(E)=\min _{u \in \operatorname{Span}\left\{\xi_{n}\right\}} \frac{\|u\|_{w, \partial \Omega}}{\|u\|_{\Omega}}=\min _{\boldsymbol{\alpha} \neq \mathbf{0}} \sqrt{\frac{\boldsymbol{\alpha}^{H} F(E) \boldsymbol{\alpha}}{\boldsymbol{\alpha}^{H} G(E) \boldsymbol{\alpha}}}=\sqrt{\hat{\lambda}_{1}(E)},
$$

where $\hat{\lambda}_{1}(E)$ is the smallest generalized eigenvalue of the matrix pencil defined by

$$
F \boldsymbol{\alpha}=\hat{\lambda} G \boldsymbol{\alpha} .
$$

Note that here, and from now on, we use the weighted boundary norm (1.7) in place of $\|u\|_{\partial \Omega}$. The matrix elements are (for $m, n=1, \ldots, N$ )

$$
F_{m n}(E):=\int_{\partial \Omega} w(s) \overline{\xi_{m}(s)} \xi_{n}(s) d s, \quad G_{m n}(E):=\left(\xi_{m}, \xi_{n}\right)_{\Omega} .
$$

With a good choice of basis set (e.g., see section 4.2$), \hat{\lambda}_{1}(E)$ is very close to its minimum achievable value $\lambda_{1}(E)$.

We now discuss how we fill $F$ and $G$ efficiently, which is not trivial at high frequency. Naively, we might expect that $N^{2}$ separate boundary integrals are required to fill $F$, where $N$ is the basis size. As we discuss below (and in [2]) to achieve reasonable approximations of eigenmodes, $N$ must scale like $\sqrt{E}$ at high frequency. Each such integral must be approximated using a set of $M$ quadrature points $\mathbf{x}_{i} \in \partial \Omega$. Since integrands oscillate on the wavelength scale, it turns out that $M$ must also scale like $\sqrt{E}$, so $M$ is the same order as $N$. Thus $O\left(N^{3}\right)$ basis function evaluations would be required to fill $F$. However, we can reduce this to $O\left(N^{2}\right)$ by writing $F \approx A^{H} W A$, where the $M \times N$ rectangular matrix $A$ has elements $A_{\text {in }}(E):=\xi_{n}\left(\mathbf{x}_{i}\right)$ and $W$ is a diagonal matrix containing the products of $w\left(\mathbf{x}_{i}\right)$ and the quadrature weights. The dense matrix-matrix product remains $O\left(N^{3}\right)$, but now the prefactor is much smaller (since basis functions are typically trigonometric or Bessels and may require $10^{2}$ flops or more per evaluation).

The naive cost of filling $G$ is worse: each domain integral would require $O\left(N^{2}\right)$ quadrature points to handle the oscillatory integrand; even using the factorization above would bring this only down to $O\left(N^{3}\right)$ basis evaluations. However, we may make use of the following identity [4, Lemma 3.1] (also see [3, Appendix H]). Let $-\Delta u=E u$ and $-\Delta v=E v$ hold in any Lipschitz domain $\Omega \in \mathbb{R}^{2}$, then

$$
(u, v)_{\Omega}=\frac{1}{2 E} \int_{\partial \Omega}(\mathbf{x} \cdot \mathbf{n})(E \bar{u} v-\nabla \bar{u} \cdot \nabla v)+(\mathbf{x} \cdot \nabla \bar{u})(\mathbf{n} \cdot \nabla v)+(\mathbf{n} \cdot \nabla \bar{u})(\mathbf{x} \cdot \nabla v) d s
$$

expresses the domain inner product as a sum of four boundary inner products. Substituting this into (4.3) using $u=\xi_{m}$ and $v=\xi_{n}$, we see that $G$ can be written as a sum of four boundary integral matrices of similar form to $F$. By computing each 
of the four terms using a product of rectangular matrices as with $F$, we may, therefore, fill $G$ using only $O\left(N^{2}\right)$ basis (and first-derivative) evaluations plus a few dense matrix-matrix products which are $O\left(N^{3}\right)$ with a small prefactor.

Solving the generalized eigenvalue problem (4.2) has an interesting twist: it turns out to become numerically singular, with $F$ and $G$ effectively acquiring a common nullspace whenever $N$ is chosen large enough to achieve high accuracy. This is due to the fact that as $N$ increases, there arise unit-norm linear combinations of basis functions which have exponentially small values in the closure of $\Omega$; this ill-conditioning of the basis appears to be a general feature of MPS type methods [12, 5, 2]. As a consequence, standard methods, such as Cholesky factorization of the (formally) positivedefinite matrix $G$ [20, section 8.7] or QZ decomposition [20, section 7.7], do not produce reliable eigenvalues of $(4.2) .{ }^{2}$ However, by using the following regularization method, we project out the numerical nullspace and accurately compute the remaining "stable" (in the sense of [15]) eigenvalues. We diagonalize the Hermitian matrix $G=$ $V D V^{H}$ (where $V$ is unitary), then define $\tilde{D}$ to be the diagonal matrix containing only the subset of entries of $D$ which exceed a cutoff $\varepsilon_{\text {reg }}$ (typically $10^{-15}$ ) times $D$ 's largest entry. Defining $\tilde{V}$ to contain as columns the corresponding subset of eigenvectors and

$$
\tilde{F}:=\tilde{D}^{-1 / 2} \tilde{V}^{H} F \tilde{V} \tilde{D}^{-1 / 2},
$$

we diagonalize $\tilde{F}=U \Lambda U^{H}$. The diagonal matrix $\Lambda$ contains approximations to the stable eigenvalues $\hat{\lambda}$ of the pencil (4.2), with eigenvectors as the columns of $\tilde{V} \tilde{D}^{-1 / 2} U$.

The above method was used by Vergini-Saraceno [46] and is similar to a more general procedure of Fix-Heiberger [15], who show that the error introduced into the stable eigenvalues is $O\left(\varepsilon_{\text {reg }}\right)$. In practice, we find that the errors are bounded by roughly $10^{3} \varepsilon_{\text {reg }}$. We believe that this prefactor reflects the closeness of the nullspaces of $F$ and $G$; however, this method deserves a full analysis, which we will not attempt here. We also find (by extrapolating in $\varepsilon_{\text {reg }}$ ) that the approximate $\hat{\lambda}_{1}$ overestimates the true value. Note that Betcke's recent GSVD method [7] includes a regularization similar to the above and can give higher accuracy; we discuss this in comment 2 of section 5. Finally, since only the lowest (or lowest few) eigenvalues are needed, one might expect that an iterative method could improve upon the above $O\left(N^{3}\right)$ effort.

4.2. Testing the applicability of the main theorem. Theorem 1.1 is an asymptotic result: given an $\epsilon>0$, it does not tell us in how large a (punctured) neighborhood of each $E_{j}$ the inequality (1.6) holds, only that some neighborhood exists. We now show that, in practice, for a small choice of $\epsilon$, the valid neighborhoods around each $E_{j}$ coalesce so that the theorem may be applied to essentially the whole positive real axis, i.e., $\Sigma_{\epsilon}=[a, \infty)$ for some small $a$. Our three test domains are defined and shown in Figure 1.2 and represent three classes of interest: (S) is smooth, (T) is a triangle with one singular $(5 \pi / 12)$ corner, and $(\mathrm{H})$ is a "horseshoe" with $C^{1}$ continuous boundary. Recall that a singular corner is a corner with angle not equal to $\pi / n$ for some $n \in \mathbb{N}[5]$. (S) and (T) are strictly star-shaped, but $(\mathrm{H})$ is not.

Before presenting results, we discuss implementation details. To compute $t_{m}(E)$, we used the method of the previous section. For $(\mathrm{S})$ and $(\mathrm{H})$, we use as basis functions $\xi_{n}$ fundamental solutions with origins (source points) placed along an exterior curve a distance of order one wavelength from $\Omega$. Such bases are efficient in many domains $[14,12]$ and show spectral convergence in analytic domains [2]. For (T), we use a cylindrical wave expansion (generalized harmonic polynomials), in polar coordinates

\footnotetext{
${ }^{2}$ We implement these with the eig command of MATLAB version 2007b.
} 

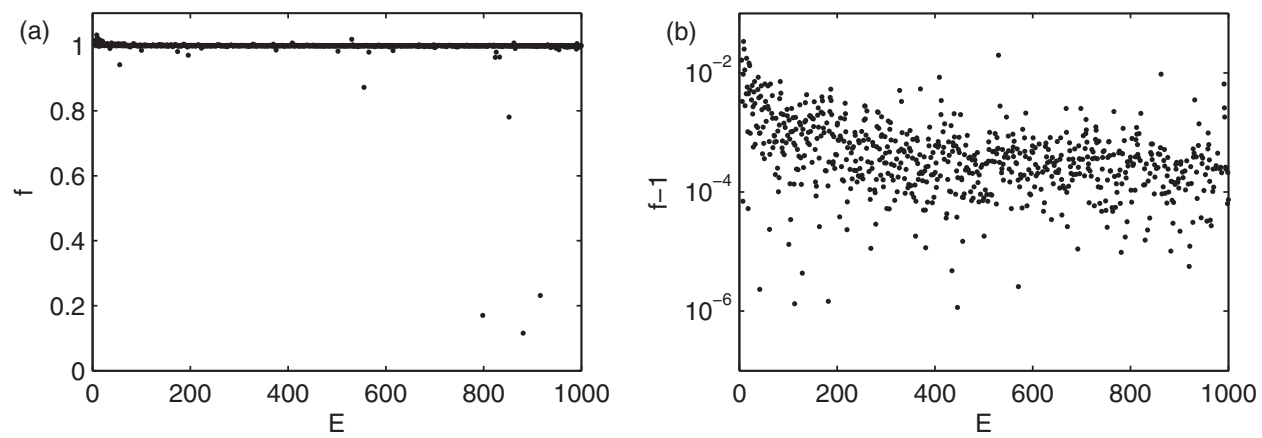

Fig. 4.1. (a) Relative slope measure $f(E)$ defined by (4.6), for the smooth domain $(S)$ with weight $w=(\mathbf{x} \cdot \mathbf{n})^{-1}$, plotted at $E=5,6,7, \ldots, 1000$. (b) Logarithmic plot of $f(E)-1$ (no point is plotted if $f(E)<1)$. The value $f(E)-1$ is the smallest value of $\epsilon$ in Theorem 1.1 such that $E \in \Sigma_{\epsilon}$.

$\xi_{n}(r, \theta)=e^{i n \theta} J_{n}(\sqrt{E} r)$ for $-N / 2 \leq n<N / 2$ and $N$ even. This basis is convergent in any bounded $\Omega$ with a finite number of corners [41, Corollary to Theorem I6.1]. It is extremely fast to evaluate (at each $r$, all $n$ values may be computed via a single Miller's downward recurrence [35, section 5.4]), and we found that it produced values of $t_{m}(E)$ for $E=E_{j}, j<10^{3}$, no larger than $10^{-3}$, adequate for our needs. (Note that this could be improved upon by adding functions adapted to the singular corner $[10,5]$.) In all three domains the scaling of basis size with wavenumber was linear: we typically chose between 3 to 6 degrees of freedom per wavelength on the boundary. Boundary quadrature was equispaced in angle for (S), and Clenshaw-Curtis [44] on each side of $(\mathrm{T})$ and each arc of $(\mathrm{H})$. In all cases, the number of quadrature points $M$ was chosen to be large enough to make the results insensitive to $M$ (as discussed in [2]); generally, $M$ was between $N$ and $2 N$. For (S) and (T), Dirichlet eigenvalues $E_{j}$ were independently located with the scaling method $[46,3,4]$ to an absolute error of roughly $10^{-3}$; for $(\mathrm{H})$, these were located by finding minima of $t_{m}(E)$. In all cases, the first two terms of Weyl's law for the asymptotics of eigenvalues [22] was used to eliminate the possibility of missing eigenvalues.

Motivated by (1.6), given some constant $C_{\Omega}$, we will study the "relative slope measure"

$$
f(E):=\frac{1}{C_{\Omega} t_{m}(E)} \min _{j} \frac{\left|E-E_{j}\right|}{\sqrt{E_{j}}} .
$$

The interpretation is that $f(E)-1$ gives a strict lower bound on $\epsilon$ such that $E \in \Sigma_{\epsilon}$. A value of $f$ close to 1 shows that the perturbative result Theorem 2.2 that we used to prove Theorem 1.1 is accurately predicting the slopes in curves such as Figure 1.1.

We start with domain $(\mathrm{S})$, and choose $w=(\mathbf{x} \cdot \mathbf{n})^{-1}$, thus use $C_{\Omega}=\sqrt{2}$. In Figure 4.1 we show $f(E)$ computed at $E=5,6,7, \ldots, 1000$. Note that the lowest 242 Dirichlet eigenvalues $E_{j}$ lie in the interval $[5,1000]$ and have no known correlation with the integers (we have checked that random sampling of $E$ gives the same behavior). It is clear that $f$ is almost always close to 1 , in fact, the largest value is $f(9)=1.0335$. Panel (b) shows the overall tendency that $f$ values approach 1 from above as $E$ grows. Occasionally, $f$ values are significantly smaller than 1: this happens only when $E$ is very close to $E_{j}$ for some $j$ and limitations of the basis set prevent $t_{m}(E)$ from reaching its theoretical minimum. Occasionally there is an anomalously large $f$ value at higher $E$ : in all cases these spikes in $f(E)$ occur for $E$ very close to $E_{j}$ for some $j$ and are due to the errors in the computed $E_{j}$. For instance, the largest such value shown is 

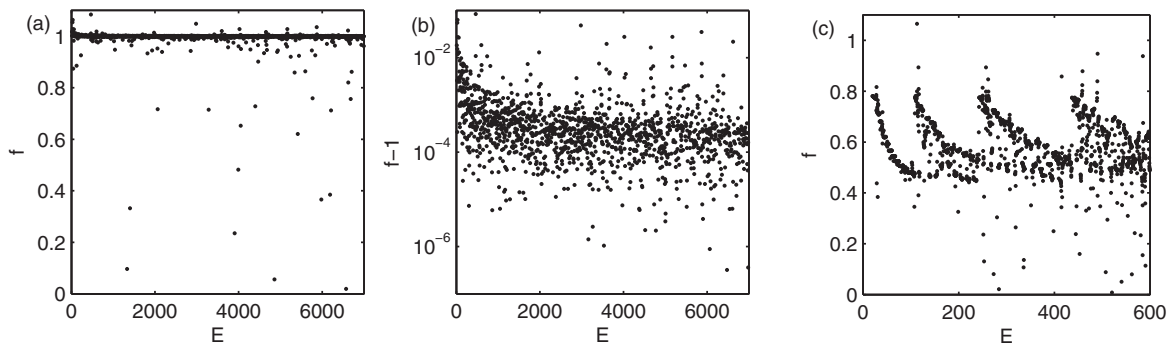

Fig. 4.2. (a,b) Same as Figure 4.1 except for the nonsmooth triangle domain $(T)$ with weight $w=(\mathbf{x} \cdot \mathbf{n})^{-1}$, plotted at $E=15,18, \ldots, 6999$. (c) Same as (a) except for horseshoe domain (H) with weight $w=1$, plotted at $E=20,20.3, \ldots, 599.9$.

$f(530)=1.0198$, where $E$ is very close to $E_{j}=530.023$. The same tendency persists at higher frequencies: computing 285 samples of $f(E)$ at $E=10007,10014, \ldots, 11995$, with $N=662, M=1277$, and using the 517 eigenvalues $E_{j}$ lying in $[10000,12000]$, we found that the largest value was $f(10511)=1.030$. This again was traced to error in the nearby eigenvalue $E_{j}=10510.768$, and all exact $f$ values are smaller than this. Thus for domain (S), there is strong evidence that a small value such as $\epsilon=0.05$ is sufficient for the theorem to hold for all parameter values $E>5$.

For the triangle $(\mathrm{T})$, we use the same weight and $C_{\Omega}$. We compute $f(E)$ at 2329 values in the interval $E=[15,6999]$, which includes the lowest 625 Dirichlet eigenvalues. As Figure 4.2(a) and (b) show, the results are essentially identical to the smooth domain: $f$ values tend to decrease towards 1 with increasing $E$. The largest value found was $f(468)=1.0840$. Finally, for the horseshoe $(H)$, which is not starshaped, we choose $w=1$ and $C_{\Omega}=10 / 3$ (see Remark 2.1). Figure 4.2(c) shows $f(E)$ computed at 1934 values in the interval $E=[20,600]$, which includes the lowest 137 Dirichlet eigenvalues. Other than the value $f(113)=1.065, f$ is always less than 1 . Periodicity in slope is evident, due to transverse mode cutoffs in the radial direction.

In conclusion, $\epsilon$ may be chosen much less than 1 , thus its exact value is inconsequential for the number of significant digits which may be claimed using Theorem 1.1.

4.3. Application to accurate inclusion of high eigenvalues. Taking domain (S) as an example, we now show how, at high eigenvalue, the bounds achievable via Theorem 1.1 improve upon those of Moler-Payne (1.4). For the constant in the latter, one has $C_{\Omega}^{\prime}=q_{1}^{-1 / 2}$, where $q_{1}$ is the lowest eigenvalue of a Stekloff eigenproblem for the domain $[28,(2.11)]$. In star-shaped domains $q_{1} \geq E_{1}^{1 / 2} r / 2 R$, where $r$ and $R$ are, respectively, the minimum and maximum of $\mathbf{x} \cdot \mathbf{n}$ on $\partial \Omega$ [28, Table I]. For domain (S), this gives $C_{\Omega}^{\prime}=1.43$. One could improve this by an $O(1)$ factor by solving the Stekloff problem, however, this would scarcely affect our conclusions.

In Figure 4.3, we show an approximate mode of domain (S) with approximate eigenvalue $E=100005.2660$, computed using $N=1666$ and $M=3690$. (Note that this and the following trial eigenvalue were found via the scaling method, although this is not relevant for our results.) Its boundary error $t \approx 1.8 \times 10^{-7}$ enables the following claims: via the Moler-Payne theorem, we have an inclusion interval for some true eigenvalue $E_{j} \in[100005.24,100005.29]$, whereas via Theorem 1.1, we get $E_{j} \in[100005.2659,100005.2661]$, i.e., a relative error of $10^{-9}$ and an improvement by 2.5 digits over Moler-Payne.

At higher frequency still $E=1000019.50$, about 300 wavelengths across the domain, we find an approximate mode with boundary error $t \approx 3 \times 10^{-5}$, using 


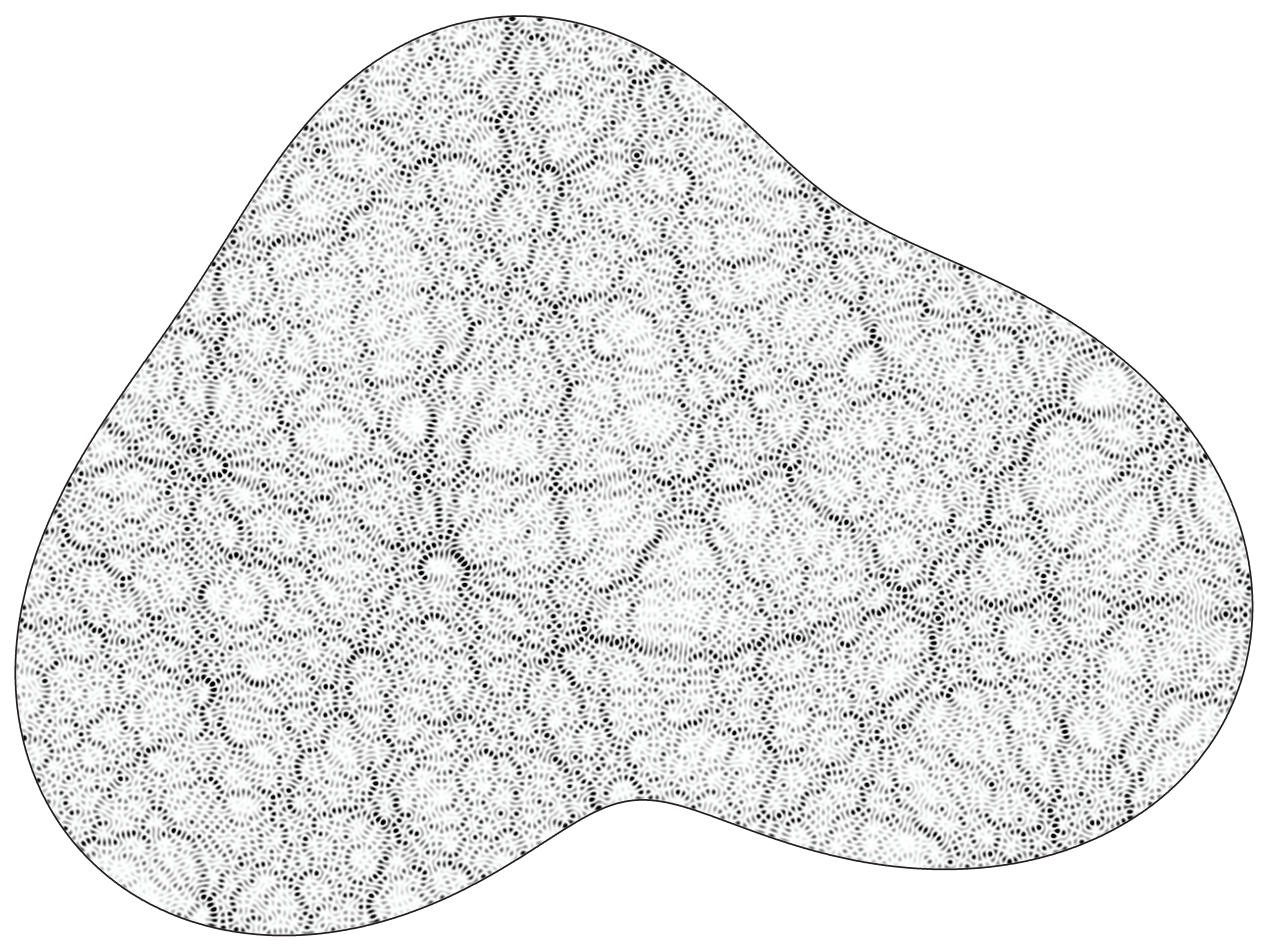

FIG. 4.3. High-lying approximate eigenmode of domain $(S)$ with eigenvalue $E_{j}$ lying in the interval [100005.2659, 100005.2661] whose bounds were computed from Theorem 1.1 with $\epsilon=0$. These bounds are 2.5 digits (320 times) better than the Moler-Payne Theorem 1.4. The intensity $\left|\phi_{j}(\mathbf{x})\right|^{2}$ is shown on a greyscale of 0 (white) to 2 (black). The mode number is $j \approx 25823$ (estimated to within $O(1)$ error using 2 terms of Weyl's asymptotic expansion [22]). There are about 100 wavelengths across the domain.

$N=M=4372$, which corresponds to only three degrees of freedom per wavelength on the boundary. (To give the reader an idea of CPU time, computation of $\hat{\lambda}_{1}$ at a single $E$ value took 33 minutes.) By Moler-Payne, we have that there is some eigenvalue $E_{j} \in[999978,1000061]$; note that this bound is scarcely useful, since it is much wider than the domain's mean eigenvalue spacing of 3.8. From our theorem we get $E_{j} \in$ [1000019.45, 1000019.54], a factor $10^{3}$ improvement in accuracy without extra effort.

How high in $E$ may one go? We note the following limitation. At $E \approx 10^{6}$, we found that the lowest eigenvalue $\hat{\lambda}_{1}$ was sensitive to $\varepsilon_{\text {reg }}$, hence we independently verified the boundary error $t$ by direct evaluation of $u$ using the generalized eigenvector coefficients. This shows that we are starting to reach the useful limit of our regularization method; see comment 2 of section 5 for a possible solution.

5. Conclusion and discussion. By analyzing the slopes of the MPS curves, we have proved a new bound (Theorem 1.1) on Dirichlet eigenvalues $E_{j}$ of smooth domains in terms of the boundary $L^{2}$ norm (1.5) of trial functions (Helmholtz solutions). This bound has better scaling at high frequency than existing bounds, improving upon them by a factor of $O(\sqrt{E})$, i.e., the wavenumber. Our main tool was analytic perturbation theory of the frequency-dependent spectrum of the natural $L^{2}(\partial \Omega)$ operator associated with (1.3) (or (2.5)); this gave us parabolic behavior at the bottom of the spectrum but limited the theorem to a (possibly small) neighborhood of each $E_{j}$. 

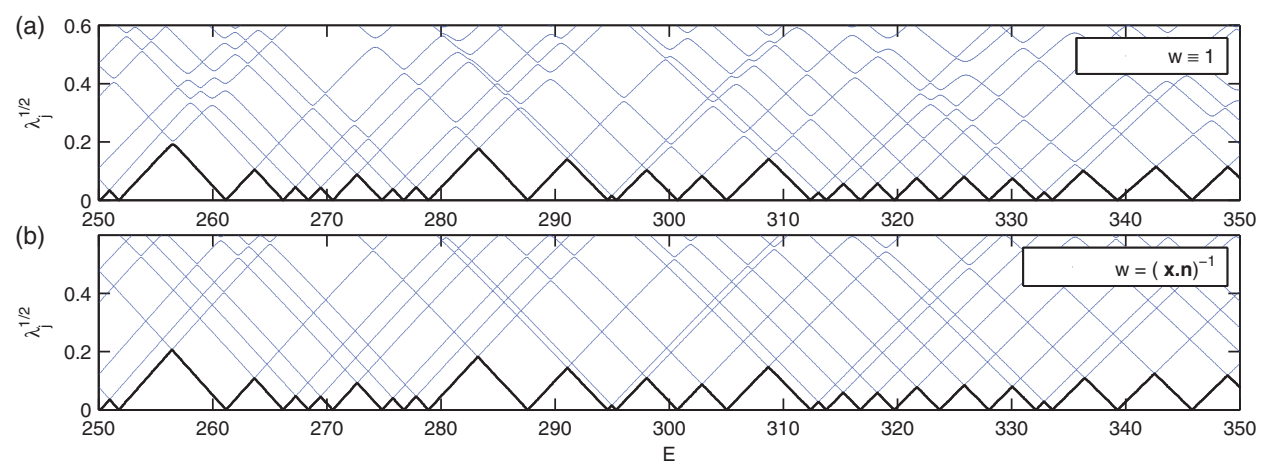

FIG. 5.1. Higher generalized eigenvalues of (4.2) (the lowest $\hat{\lambda}_{1}(E)$ is shown as a thick line) in domain (S), with weight function (a) constant $w=1$, (b) special weight $w=(\mathbf{x} \cdot \mathbf{n})^{-1}$. Observe that the slight mode-to-mode variations in slope and "avoided crossings" in (a) nearly vanish in (b).

We provided strong numerical evidence that, in fact, these neighborhoods are large; thus we believe that, in practice, the theorem may be used with confidence for all $E \in[a, \infty)$ for some $a<E_{1}$, given a small choice of $\epsilon$ such as $10^{-1}$. This we checked at thousands of $E$ values for smooth, polygonal and $C^{1}$ domains. We also presented an efficient implementation of the MPS, which involves boundary evaluations alone, and we demonstrated a 3 -digit accuracy gain via our theorem at very high frequencies.

We end with several open questions and research directions of interest.

1. We suspect Theorem 1.1 could be extended to Lipschitz domains (either via proving compactness of $A(E)$ or by taking into account a possible essential spectrum in section 3.2). Another natural next step is to seek corresponding bounds on eigenfunction error $\left\|u-\phi_{j}\right\|_{L^{2}(\Omega)}$ (as in [33, 29]).

2. Since the boundary error norm $t_{m}(E)$ is the square-root of a generalized eigenvalue, our method cannot access $t$ values below roughly $\sqrt{\varepsilon_{\text {reg }}}$, which must exceed $\sqrt{\varepsilon_{\text {mach }}} \approx 10^{-8}$ in double-precision. Betcke's GSVD method [7], by working directly with $A$ from section 4.1, avoids matrix squaring so is able to reach $t$ of order $\varepsilon_{\text {mach }}$, but requires estimation via interior points. It would be valuable to seek an efficient way to construct a "square-root" of $G$ using the boundary alone, possibly via (4.4).

3. Still [42, Theorem 4] has applied the Kato-Temple inequality to the MPS, effectively replacing $t[u]$ in (1.4) by $t[u]^{2}$, which is clearly advantageous at small $t$. The disadvantage is that a PDE must be solved; however, its utility should be compared to that of Theorem 1.1.

4. To understand why the quadratic approximation (2.10) predicts MPS eigenvalues so well, we need the 4th- and higher-order terms, via a perturbation expansion. In a similar vein, the higher generalized eigenvalues, shown by Figure 5.1, contain much tantalizing and potentially useful structure and so should be analyzed. This might lead to bounds on multiple close eigenvalues, as in recent work of Eisenstat [11].

5. Given a Lipschitz domain, how should an optimal value for $C_{w, \Omega}$ be computed for Lemma 2.3 ?

6. We have shown that the MPS is a powerful tool for high-lying Dirichlet eigenvalues. However, locating eigenvalues one by one by minimizing $t_{m}(E)$ is cumbersome, needing many evaluations of this function, each of effort $O\left(N^{3}\right)$, per eigenvalue found. The scaling method $[46,3,4]$ for star-shaped domains is 
$O(N)$ times more efficient but has no rigorous error analysis. We intend to apply tools of this paper to the scaling method.

Appendix A. Proof of analyticity of $\boldsymbol{A}_{j}(\boldsymbol{E})$, Lemma 3.2. Each term in (3.11) is analytic in the neighborhood of $E_{j}$, but we need to show that their infinite sum is also analytic. Let $R:=\frac{1}{2} \min _{m \notin[j, j+p-1]}\left|E_{m}-E_{j}\right|$ be the radius of a closed disc in the complex $E$ plane centered at $E_{j}$. Its radius is half the distance to the nearest neighboring eigenvalue; see Figure 3.1(a). Then for each $m \notin[j, j+p-1]$ and $E$ with $\left|E-E_{j}\right| \leq R$, we have $\left|E-E_{m}\right| \geq \frac{1}{3}\left|R+E_{j}-E_{m}\right|$. Using this we have for any $f \in L^{2}(\partial \Omega)$ and $\left|E-E_{j}\right| \leq R$,

$$
\begin{aligned}
\left|\left\langle f, A_{j}(E) f\right\rangle\right| & =\left|\sum_{m \notin[j, j+p-1]} \frac{\left|\left\langle f, \psi_{m}\right\rangle\right|^{2}}{\left(E-E_{m}\right)^{2}}\right| \leq 9 \sum_{m \notin[j, j+p-1]} \frac{\left|\left\langle f, \psi_{m}\right\rangle\right|^{2}}{\left(R+E_{j}-E_{m}\right)^{2}} \\
& =9\left\langle f, A_{j}\left(E_{j}+R\right) f\right\rangle \leq C\|f\|_{L^{2}(\partial \Omega)}^{2},
\end{aligned}
$$

since $E_{j}+R \notin \sigma_{D}$, thus $A$ is bounded and from (3.9), $A_{j}$ differs from $A$ by a bounded operator. For each integer $N \geq j+p$, we may define the sum

$$
A_{j}^{(N)}(E):=\left(\sum_{m=1}^{j-1}+\sum_{m=j+p}^{N}\right) \frac{\psi_{m}\left\langle\psi_{m}, \cdot\right\rangle}{\left(E-E_{m}\right)^{2}}
$$

so that $A_{j}(E)=\lim _{N \rightarrow \infty} A_{j}^{(N)}(E)$. For each $f \in L^{2}(\partial \Omega)$ and each $N$, since this sum is finite and its poles lie a distance at least $2 R$ from $E_{j}$, the Cauchy integral formula holds inside the disc:

$$
\left\langle f, A_{j}^{(N)}(E) f\right\rangle=\frac{1}{2 \pi i} \oint_{\left|z-E_{j}\right|=R} \frac{\left\langle f, A_{j}^{(N)}(z) f\right\rangle}{z-E} d z \quad \text { for }\left|E-E_{j}\right|<R .
$$

Taking the limit $N \rightarrow \infty$, we may apply the dominated convergence theorem to the right-hand side since the integrand is bounded by $\left|\left\langle f, A_{j}(z) f\right\rangle\right| /\left(R-\left|E-E_{j}\right|\right)$ and (A.1) holds. This gives, for each $f \in L^{2}(\partial \Omega)$,

$$
\left\langle f, A_{j}(E) f\right\rangle=\frac{1}{2 \pi i} \oint_{\left|z-E_{j}\right|=R} \frac{\left\langle f, A_{j}(z) f\right\rangle}{z-E} d z \quad \text { for }\left|E-E_{j}\right|<R,
$$

which proves that $\left\langle f, A_{j}(E) f\right\rangle$ is an analytic function of $E$ with a radius of convergence about $E_{j}$ of at least $R$. Using the polarization identity in complex Hilbert space (A.5)

$4\left\langle f, A_{j} g\right\rangle=\left\langle f+g, A_{j}(f+g)\right\rangle-\left\langle f-g, A_{j}(f-g)\right\rangle-i\left\langle f+i g, A_{j}(f+i g)\right\rangle+i\left\langle f-i g, A_{j}(f-i g)\right\rangle$,

we see that $\left\langle f, A_{j}(E) g\right\rangle$ is analytic for all $f, g \in L^{2}(\partial \Omega)$ (weak analyticity). By [37, Theorem VI.4, p. 189], this implies $A_{j}(E) f$ is analytic for each $f \in L^{2}(\partial \Omega)$, which is the condition required to prove the lemma.

Appendix B. Proof of Cauchy interlacing, Lemma 3.4.

The $n$th eigenvalue of $S$ has the max-min characterization [36, Theorem XIII.1]

$$
\sigma_{n}=\sup _{U_{1}, \ldots, U_{n-1}} \inf _{\substack{V \perp \operatorname{Span}\left\{U_{1}, \ldots, U_{n-1}\right\} \\\|V\|=1}}\langle V, S V\rangle,
$$


where the sup is over all possible sets of $n-1$ vectors in the Hilbert space. The analogous formula for $\tau_{n}$ is found by replacing $S$ with $R:=S-\psi\langle\psi, \cdot\rangle$. Since for all $V \in L^{2}(\partial \Omega)$ it holds that $\langle V, R V\rangle=\langle V, S V\rangle-|\langle V, \psi\rangle|^{2} \leq\langle V, S V\rangle$, the same holds for the max-min values, hence for each $n=1,2, \ldots$, we have $\tau_{n} \leq \sigma_{n}$. In addition, using (B.1) we have the inequalities

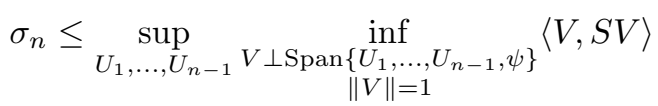

$$
\begin{aligned}
& =\sup _{U_{1}, \ldots, U_{n-1}} \inf _{V \perp \operatorname{Span}\left\{U_{1}, \ldots, U_{n-1}, \psi\right\}}\langle V, R V\rangle
\end{aligned}
$$

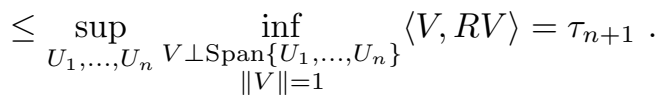

The middle equality follows since $V$ is orthogonal to $\psi$; the inequalities are standard linear subspace arguments. Thus the interlacing $\tau_{n} \leq \sigma_{n} \leq \tau_{n+1}$ is proved.

Acknowledgments. The author is indebted to Percy Deift and Jonathan Goodman for introducing many of the ideas which made this work possible. The author also thanks Ross Barnett, Timo Betcke, Leslie Greengard, Andrew Hassell, Chris Judge, Bob Kohn, Peter Lax, Kevin Lin, Mikhail Shubin, Nick Trefethen, Eduardo Vergini, and the referees for useful input.

\section{REFERENCES}

[1] A. BÄCKER, Numerical aspects of eigenvalue and eigenfunction computations for chaotic quantum systems, in The Mathematical Aspects of Quantum Maps, Lect. Notes Phys. 618, Springer, Berlin, 2003, pp. 91-144.

[2] A. H. Barnett And T. Betcke, Stability and convergence of the method of fundamental solutions for Helmholtz problems on analytic domains, J. Comput. Phys., 227 (2008), pp. 7003-7026.

[3] A. H. Barnett, Dissipation in Deforming Chaotic Billiards, Ph.D. thesis, Harvard University, Cambridge, MA, 2000, http://www.math.dartmouth.edu/ ahb/thesis_html/.

[4] A. H. Barnett, Asymptotic rate of quantum ergodicity in chaotic Euclidean billiards, Comm. Pure Appl. Math., 59 (2006), pp. 1457-88.

[5] T. Betcke and L. N. Trefethen, Reviving the method of particular solutions, SIAM Rev., 47 (2005), pp. 469-491.

[6] T. Betcke, A GSVD formulation of a domain decomposition method for planar eigenvalue problems, IMA J. Numer. Anal., 27 (2007), pp. 451-478.

[7] T. BETCKE, The generalized singular value decomposition and the method of particular solutions, SIAM J. Sci. Comput., 30 (2008), pp. 1278-1295.

[8] J. Descloux And M. Tolley, An accurate algorithm for computing the eigenvalues of a polygonal membrane, Comput. Methods Appl. Mech. Engrg., 39 (1983), pp. 37-53.

[9] T. A. Driscoll, Eigenmodes of isospectral drums, SIAM Rev., 39 (1997), pp. 1-17.

[10] S. C. Eisenstat, On the rate of convergence of the Bergman-Vekua method for the numerical solution of elliptic boundary value problems, SIAM J. Numer. Anal., 11 (1974), pp. 654680.

[11] S. C. Eisenstat, Bounds for Eigenvalues and Eigenvectors of Self-adjoint Operators, preprint, 2006.

[12] R. Ennenbach And H. Niemeyer, The inclusion of Dirichlet eigenvalues with singularity functions, Z. Angew. Math. Mech., 76 (1996), pp. 377-383.

[13] L. C. Evans, Partial Differential Equations, Grad. Stud. Math. 19, American Mathematical Society, Providence, RI, 1998.

[14] G. Fairweather and A. Karageorghis, The method of fundamental solutions for elliptic boundary value problems, Adv. Comput. Math., 9 (1998), pp. 69-95.

[15] G. Fix And R. Heiberger, An algorithm for the ill-conditioned generalized eigenvalue problem, SIAM J. Numer. Anal., 9 (1972), pp. 78-88. 
[16] L. Fox, P. Henrici, And C. Moler, Approximations and bounds for eigenvalues of elliptic operators, SIAM J. Numer. Anal., 4 (1967), pp. 89-102.

[17] L. Friedlander, Some inequalities between Dirichlet and Neumann eigenvalues, Arch. Ration. Mech. Anal., 116 (1991), pp. 153-160.

[18] P. R. Garabedian, Partial Differential Equations, John Wiley \& Sons, New York, 1964.

[19] P. GÉrard And E. Leichtnam, Ergodic properties of eigenfunctions for the Dirichlet problem, Duke Math. J., 71 (1993), pp. 559-607.

[20] G. H. Golub and C. F. Van Loan, Matrix computations, 3rd ed., Johns Hopkins Studies in the Mathematical Sciences, Johns Hopkins University Press, Baltimore, MD, 1996.

[21] C. Gordon, D. WebB, And S. Wolpert, Isospectral plane domains and surfaces via Riemannian orbifolds, Invent. Math., 110 (1992), pp. 1-22.

[22] M. C. Gutzwiller, Chaos in Classical and Quantum Mechanics, Interdiscip. Appl. Math., Springer, New York, 1990.

[23] A. HASSELL, personal communication, 2005.

[24] A. Hassell and T. TAO, Upper and lower bounds for normal derivatives of Dirichlet eigenfunctions, Math. Res. Lett., 9 (2002), pp. 289-305.

[25] E. J. Heller, Bound-state eigenfunctions of classically chaotic Hamiltonian systems: Scars of periodic orbits, Phys. Rev. Lett., 53 (1984), pp. 1515-1518.

[26] E. J. Heller, Wavepacket dynamics and quantum chaology, in Chaos et Physique Quantique (Les Houches, 1989), North-Holland, Amsterdam, 1991, pp. 547-664.

[27] A. KARAgEORGHis, The method of fundamental solutions for the calculation of the eigenvalues of the Helmholtz equation, Appl. Math. Lett., 14 (2001), pp. 837-842.

[28] J. R. Kuttler and V. G. Sigillito, Inequalities for membrane and Stekloff eigenvalues, J. Math. Anal. Appl., 23 (1968), pp. 148-160.

[29] J. R. Kuttler and V. G. Sigillito, Bounding eigenvalues of elliptic operators, SIAM J. Math. Anal., 9 (1978), pp. 768-778.

[30] J. R. Kuttler And V. G. Sigillito, Eigenvalues of the Laplacian in two dimensions, SIAM Rev., 26 (1984), pp. 163-193.

[31] J.-L. Lions and E. Magenes, Problèmes aux limites non homogènes et applications, Vol. 3, Dunod, Paris, 1970. Travaux et Recherches Mathématiques, 20.

[32] W. C. H. McLean, Strongly Elliptic Systems and Boundary Integral Equations, Cambridge University Press, London, 2000.

[33] C. B. Moler And L. E. PAYne, Bounds for eigenvalues and eigenvectors of symmetric operators, SIAM J. Numer. Anal., 5 (1968), pp. 64-70.

[34] A. Nachman, J. Sylvester, And G. Uhlmann, An n-dimensional Borg-Levinson theorem, Comm. Math. Phys., 115 (1988), pp. 595-605.

[35] W. H. Press, S. A. Teukolsky, W. T. Vetterling, and B. P. Flannery, Numerical Recipes in $C$, 2nd ed., Cambridge University Press, Cambridge, 2002.

[36] M. Reed And B. Simon, Methods of Modern Mathematical Physics. IV. Analysis of Operators, Academic Press, New York, 1978.

[37] M. Reed And B. Simon, Methods of Modern Mathematical Physics. I. Functional Analysis, 2nd ed., Academic Press, New York, 1980.

[38] F. Rellich, Darstellung der Eigenwerte von $\Delta u+\lambda u=0$ durch ein Randintegral, Math. Z., 46 (1940), pp. 635-636.

[39] F. Rellich, Perturbation Theory of Eigenvalue Problems, Gordon and Breach, New York, 1969.

[40] N. SAITO, Data analysis and representation on a general domain using eigenfunctions of Laplacian, Appl. Comput. Harmon. Anal., 25 (2008), pp. 68-97.

[41] N. L. SCHRYER, Constructive approximation of solutions to linear elliptic boundary value problems, SIAM J. Numer. Anal., 9 (1972), pp. 546-572.

[42] G. Still, Computable bounds for eigenvalues and eigenfunctions of elliptic differential operators, Numer. Math., 54 (1988), pp. 201-223.

[43] L. N. Trefethen and T. Betcke, Computed eigenmodes of planar regions, Contemp. Math., 412 (2006), pp. 297-314.

[44] L. N. Trefethen, Spectral Methods in MATLAB, Vol. 10, Software, Environments, and Tools, SIAM, Philadelphia, PA, 2000.

[45] H. E. Tureci, H. G. L. Schwefel, P. Jacquod, And A. D. Stone, Modes of wave-chaotic dielectric resonators, Prog. Optics, 47 (2005), pp. 75-137.

[46] E. Vergini and M. Saraceno, Calculation by scaling of highly excited states of billiards, Phys. Rev. E, 52 (1995), pp. 2204-2207.

[47] S. Zelditch, Quantum ergodicity and mixing of eigenfunctions, in Elsevier Encyclopedia of Mathematical Physics, Vol. 1, Academic Press/Elsevier Science, Oxford, 2006, pp. 183-196, arXiv:math-ph/0503026. 